\title{
Controversias sobre el uso de bloqueadores neuromusculares en el síndrome de insuficiencia respiratoria aguda
}

\author{
Idoris Cordero-Escobar ${ }^{1 *}$ y Caridad de Dios-Soler-Morejón ${ }^{2}$ \\ ${ }^{1}$ Servicio de Anestesiología y Reanimación; ${ }^{2}$ Medicina Intensiva y Emergencias. Hospital Clínico Quirúrgico Hermanos Ameijeiras, Ciudad de La \\ Habana, Cuba
}

\begin{abstract}
Resumen
Los bloqueadores neuromusculares son fármacos que se utilizan como complemento de la anestesia general. Actualizar revisión de la literatura sobre el uso de bloqueadores neuromusculares en el síndrome de insuficiencia respiratoria aguda. La posibilidad de uso en este contexto ha abierto un vasto campo de investigación. Su administración prolongada se ha asociado a complicaciones $y$, a pesar de las controversias existentes sobre su empleo, en años recientes se ha observado que puede considerarse un fármaco adyuvante para el tratamiento de este síndrome. Se requiere aumentar las evidencias científicas para garantizar un elevado perfil de seguridad de estos fármacos como adyuvantes en el tratamiento del síndrome de insuficiencia respiratoria aguda antes que se pueda recomendar su uso sistemático. A la luz de los conocimientos actuales y lo controvertido del tema, la indicación de estos fármacos se debe individualizar en el paciente crítico en general y en aquel con síndrome de insuficiencia respiratoria aguda en especial.
\end{abstract}

Palabras clave: Bloqueadores neuromusculares. Insuficiencia respiratoria aguda. Receptores acetilcolínicos. Complicaciones. Mortalidad.

\section{Controversies about the use of neuromuscular blockers in acute respiratory failure syndrome}

\begin{abstract}
Neuromuscular blockers are drugs that are used as a complement to general anesthesia. To update review of the literature on the use of neuromuscular blockers in acute respiratory failure syndrome. The possibility of use in this context has opened a vast field of research. Its prolonged administration has been associated with complications and despite the controversies about its use, in recent years it has been observed that it can be considered an adjuvant drug for the treatment of this syndrome. It is necessary to increase the scientific evidence to guarantee a high safety profile of these drugs as adjuvants in the treatment of acute respiratory failure syndrome before their systematic use can be recommended. In light of current knowledge and the controversy of the subject, the indication of these drugs should be individualized in the critical patient in general and in that with acute respiratory distress syndrome in particular.
\end{abstract}

Key words: Neuromuscular blockers. Acute respiratory failure. Acetylcholine receptors. Complications. Mortality.

Correspondencia:

*Idoris Cordero-Escobar

E-mail: ice@infomed.sld.cu
Disponible en internet: 22-11-2019 Rev Argent Anestesiol. 2019;77(1):13-17 www.revistaargentinaanestesiologia.com 0370-7792 @ 2019 Federación Argentina de Asociaciones, Anestesia, Analgesia y Reanimación. Publicado por Permanyer México SA de CV. Este es un artículo open access bajo la licencia CC BY-NC-ND (http://creativecommons.org/licenses/by-nc-nd/4.0/). 


\section{Introducción}

Los bloqueadores neuromusculares (BNM) son fármacos que se emplean, a pesar de sus efectos adversos, como complemento de la anestesia general, ya que cuando son administrados durante corto tiempo, se aprovechan sus propiedades para la realización de diversas técnicas quirúrgicas y anestésicas.

Entre sus efectos ventajosos se pueden citar: la relajación de las cuerdas vocales para facilitar la laringoscopia y la intubación, lo que permite proveer una oxigenación y ventilación mecánica adecuada, así como la relajación de la musculatura estriada para proporcionar una intervención quirúrgica menos compleja para el cirujano, por solo mencionar algunas.

Sin embargo, en el paciente crítico, el uso de los BNM es un tema controvertido. En la década de 1970 del siglo XX, un gran número de pacientes ventilados requerían su uso para acoplarlos al ventilador artificial o mantener la sincronía con el mismo. En esa época, el BNM más utilizado en EE.UU. era el vecuronio, mientras que en el Reino Unido era el atracurio ${ }^{1-3}$.

A mediados de la década siguiente, con el advenimiento de los BNM de acción intermedia, se hizo más frecuente su uso, por la posibilidad de administrarlos en infusión continua, pues así se podía mantener el bloqueo neuromuscular por largos periodos, se minimizaban los efectos hemodinámicos y las fluctuaciones derivadas de los rápidos cambios de concentración de estos fármacos, como ocurría con la administración intermitente $^{3-5}$. El gran margen de seguridad de los nuevos BNM hizo que esta práctica se generalizara para una gran variedad de indicaciones ${ }^{2}$.

A comienzos de la década de 1990 del siglo XX, se realizaron estudios prospectivos que informaron que hasta el $70 \%$ de los pacientes críticos podían tener debilidad muscular después de una prolongada administración de vecuronio o pancuronio, lo que hizo finalmente limitar sus indicaciones y disminuir al mínimo indispensable los periodos y la profundidad del bloqueo $0^{1,3,6-11}$.

Desde 1977, fue cuando comenzaron a publicarse alarmantes observaciones en pacientes asmáticos que con el uso de BNM de tipo esteroideo desarrollaron una debilidad muscular progresiva adquirida a la que se le denominó miopatía grave1,2,5,12. Esta complicación tiene implicaciones graves en el paciente crítico e influye negativamente en el proceso de deshabituación del ventilador y, por tanto, en su recuperación y pronóstico vital ${ }^{3}$.

Cuando en 1997, Khuenl-Brady ${ }^{3}$ publicó los efectos que la aplicación de atracurio produjo en un grupo de pacientes críticos que evolucionaron a este mismo cuadro clínico, se desató una controversia sobre cuáles eran los mecanismos patogénicos de esta entidad, ya que el atracurio pertenece a otro grupo farmacológico y se categoriza como benzilisoquinolineo.

Sin embargo, en los últimos años se han obtenido algunas pruebas que sugieren efectos favorables del empleo de estos fármacos en el tratamiento del síndrome de insuficiencia respiratoria aguda (SIRA).

El objetivo de este artículo es realizar una revisión de la literatura sobre el uso de BNM en el tratamiento de los pacientes críticos con síndrome de distrés respiratorio.

\section{Desarrollo}

Las opiniones respecto al uso de los BNM aún son controvertidas en la comunidad científica ${ }^{11-37}$. En una revisión realizada por las autoras de este estudio en la base de datos bibliográfica PubMed, con los descriptores muscle relaxants AND acute respiratory distress syndrome, en los últimos 5 años se localizaron 40 artículos. De ellos, 17 son revisiones sistemáticas sobre el tema.

\section{Bloqueadores neuromusculares: NO}

Los pacientes en estado crítico ingresados en la unidad de cuidados intensivos (UCI) desarrollan a menudo una debilidad adquirida, aguda o subaguda, que se caracteriza por su inicio difuso en las extremidades y se asocia a factores como la ventilación mecánica prolongada, mayor estancia en la $\mathrm{UCl}$ e incremento de la mortalidad $^{6}$. Esta debilidad puede llegar a ser grave y persistente ${ }^{13-18}$ porque compromete tanto al sistema nervioso periférico como los músculos ${ }^{13}$, por lo que algunos autores recomiendan la sedación profunda ${ }^{16}$, mientras aseveran que el uso de BNM podría estar asociado de forma independiente con una mayor mortalidad ${ }^{16,17}$.

Al respecto, Howard, et al. ${ }^{13}$ plantearon que la disfunción de la musculatura respiratoria, particularmente del diafragma, causada por esta miopatía, puede jugar un papel importante en los mecanismos fisiopatológicos que conducen a dificultad en el destete en pacientes ventilados mecánicamente.

Las pruebas a favor que los BNM estén implicados en la producción de la llamada miopatía grave del paciente crítico, debilidad o parálisis generalizada adquirida del paciente crítico o polineuropatía del paciente crítico son recurrentes en la literatura revisada ${ }^{1,4,5,7,12,15,20-25}$. Entre los factores implicados se destaca el uso prolongado de los BNM. 
Los mecanismos etiopatogénicos que se plantean son variados. Según Khuenl-Brady ${ }^{3}$, es probable que la utilización prolongada y continua de BNM determine cambios pre, postsinápticos, o ambos, que no son predecibles, independientemente de su mecanismo de acción. Se considera que a nivel presináptico, los BNM causan interferencia con la corriente iónica de calcio en la terminal nerviosa, inhiben la liberación de acetilcolina y disminuyen la síntesis del receptor acetilcolínico por un mecanismo de sobreexposición denominado up regulation, lo que determina aumento de la sensibilidad al bloqueo. Por su parte, la exposición prolongada del receptor postsináptico a un agonista, resulta en la síntesis incrementada del receptor (mecanismo de down regulation) ${ }^{3}$. Como los nuevos receptores son inmaduros, muestran sensibilidad aumentada a la acetilcolina y disminuida a los BNM. Tales efectos pueden influir gradualmente en el aumento o la disminución de la efectividad de estos fármacos, en dependencia de la naturaleza de los cambios a largo plazo.

Otros autores sugieren que el desarrollo de dicha entidad parece guardar relación con una profunda «denervación química» del músculo con aumento de la creatincinasa plasmática. En estos pacientes la biopsia muscular ha evidenciado atrofia y degeneración de las miofibrillas 3,10,15-21.

Según el criterio de Weber-Carstens, et al. ${ }^{14}$, la inflamación sistémica que ocurre durante los primeros momentos de la enfermedad crítica podría ser el principal factor de riesgo para el desarrollo de la miopatía grave. Estos autores consideran que el deterioro inducido por la inflamación y el factor de crecimiento mediado por la sensibilidad a la insulina son los que determinan el desarrollo de esta entidad.

Otra hipótesis que intenta explicar la etiopatogenia de esta entidad plantea que en los pacientes críticos se producen trastornos de la microcirculación. La hipoxia axonal causa edema endoneural y hace más vulnerable al sistema nervioso periférico 22-30.

Además, los pacientes críticamente enfermos están expuestos a un riesgo elevado de aparición de alteraciones de las propiedades farmacocinéticas y farmacodinámicas de los BNM. Además, la utilización concurrente de otros fármacos puede alterar la respuesta normal de la unión neuromuscular a la acetilcolina o modificar la capacidad del organismo de distribuir y eliminar el fármaco o sus metabolitos ${ }^{2,5,13-18}$.

\section{Bloqueadores neuromusculares: Sí}

A pesar de las desventajas antes mencionadas, el interés en el papel de los BNM en el tratamiento del
SIRA se ha renovado desde que un reciente ensayo clínico aleatorizado mostró una reducción en la mortalidad asociada con su uso ${ }^{30}$.

Recientemente, en un editorial titulado Acetylcholine receptor antagonists in acute respiratory distress syndrome: much more tan muscle relaxants, basado en la investigación de Sottile, et al. ${ }^{31}$, quienes concluyeron que el uso de BNM se asocia con una disminución de los biomarcadores de la lesión pulmonar epitelial, endotelial y de inflamación sistémica en pacientes con SIRA que recibieron ventilación con un volumen tidal bajo, con una relación $\mathrm{PO}_{2} / \mathrm{FiO}_{2} \leq 120 \mathrm{mmHg}$.

Forel, et al. ${ }^{8}$ confirmaron que cuando los BNM se administraron de forma temprana en pacientes con SIRA se asociaron a una menor mortalidad ajustada a los 90 días, una morbilidad disminuida, disminución de los días de ventilación mecánica, menor estadía y menos días con insuficiencia de órganos. Estos beneficios se lograron sin aumentar la incidencia de debilidad muscular adquirida en la $\mathrm{UCl}$.

Un año después (2010), Papazian, et al. ${ }^{7}$ publicaron los resultados de un ensayo clínico controlado, aleatorizado y multicéntrico (estudio ACURASYS) en el que se demostró que el cisatracurio utilizado precozmente en infusión continua durante 48 h, redujo 90 días la mortalidad y mejoró la oxigenación en pacientes con SIRA o con una relación $\mathrm{PaO}_{2} / \mathrm{FiO}_{2} \leq 120 \mathrm{mmHg}$.

El mecanismo por el cual esto sucede no está claro, pero probablemente involucre una combinación de factores que incluye la reducción del consumo de oxígeno, disminución del gasto cardíaco, del flujo sanguíneo pulmonar, así como efectos directos antiinflamatorios de los BNM; pero el mecanismo más significativo es muy probablemente la supresión del esfuerzo respiratorio del paciente, que limita el riesgo de colapso alveolar y la sobredistensión pulmonar ${ }^{9,10,26}$, mejora la sincronía paciente-ventilador (disminuye el consumo de oxígeno) y la respuesta inflamatoria sistémica asociada al SIRA ${ }^{27}$.

Se ha afirmado que cuando los BNM se utilizan en el SIRA en etapas tempranas pueden ayudar a establecer una estrategia de protección pulmonar. Dicha estrategia conduce a mejorar la oxigenación, disminuir la inflamación pulmonar y sistémica, con mejoría potencial de la mortalidad, sin olvidar que estos fármacos pueden producir miopatía o polineuropatía del paciente crítico, sobre todo cuando se utilizan en periodos prolongados $^{15,31-37}$.

Aunque se ha expresado que la administración temprana de BNM mejora la supervivencia ajustada en pacientes con lesión pulmonar grave, algunos autores 
afirman que estos resultados podrían inducir a un mayor uso de estos fármacos. Si bien los datos emergentes apoyan que el cisatracurio en pacientes con SIRA debe ser el de elección, la literatura actual no aconseja el uso de BNM para otras indicaciones en pacientes críticos ${ }^{14}$.

Los BNM aminoesteroideos se pueden considerar fármacos adyuvantes con función protectora del pulmón en los inicios del SIRA, y una alternativa en el shock séptico con lesión pulmonar menos grave, aunque según Gorson, et al..$^{15}$, las conclusiones definitivas precisan de más estudios.

Como proponen varios autores ${ }^{20,21}$, a partir de los recientes avances en la monitorización de la función neuromuscular y la existencia de nuevos agentes BNM se hace necesario la actualización en el uso de estos fármacos en el paciente crítico. Los BNM deben elegirse de acuerdo a sus propiedades farmacocinéticas y la fisiopatología del paciente crítico. Aquellos fármacos, como los bencilisoquinolineos, con metabolismo dependiente de la hidrólisis de Hoffman, así como los que tienen un rápido inicio de acción, deben ser los de elección en estos enfermos, y sustituir a aquellos que tienen metabolismo hepático o biliar.

Se concluye que la posibilidad del uso de los BNM en este contexto ha abierto un vasto campo de investigación. Se requiere aumentar las evidencias científicas en cantidad y calidad y garantizar un elevado perfil de seguridad para la aplicación de estos fármacos como adyuvantes en el tratamiento del SIRA antes que se pueda recomendar su uso sistemático. A la luz de los conocimientos actuales y lo controvertido del tema, a criterio de las autoras, la indicación de BNM debe individualizarse en el paciente crítico en general y en aquel con SIRA en especial.

\section{Conflicto de intereses}

Los autores declaran que no existe conflicto de intereses

\section{Responsabilidades éticas}

Protección de personas y animales. Los autores declaran que para esta investigación no se han realizado experimentos en seres humanos ni en animales.

Confidencialidad de los datos. Los autores declaran que en este artículo no aparecen datos de pacientes.

Derecho a la privacidad y consentimiento informado. Los autores declaran que en este artículo no aparecen datos de pacientes.

\section{Bibliografía}

1. Bustamante BR. Bloqueadores Neuromusculares en Cuidados Intensivos. Rev Chil Anest. 2004;33(2):147-74.

2. Alí HH, Miller RD. Monitorización de la función neuromuscular. En: Mi-

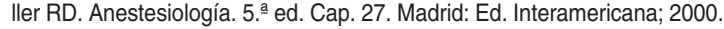
p. 827-72.

3. Khuenl-Brady KS. Pathophysiological changes of the neuromuscular transmission in the critically ill patient. Acta Anaesthesiol Scand. 1997; 11:100-8.

4. Cordero Escobar I. Relajantes musculares en el paciente crítico. En: Cordero Escobar I. Relajantes musculares en la práctica anestesiológica. Cap. 11. La Habana: ECIMED; 2010. p. 121-6.

5. Takei T. Intensive Care Unit-acquired Weakness: Development of Polyneuropathy and Myopathy in Critically III Patients. Brain Nerve. 2014; 66(2):161-70

6. Jansen D, de Vries $\mathrm{H}$, Leo M, Heunks A. Antagonistas del receptor de acetilcolina en pacientes agudos síndrome de dificultad respiratoria: mucho más que los relajantes musculares. Critical Care. 2018;22:132.

7. Papazian L, Forel JM, Gacouin A, Penot-Ragon C, Perrin G, Loundou A, et al. Neuromuscular blockers in early acute respiratory distress syndrome. N Engl J Med. 2010;363(12):1107-16.

8. Forel JM, Roch A, Papazian L. Paralytics in critical care: not always the bad guy. Curr Opin Crit Care. 2009;15(1):59-66.

9. Puthucheary Z, Rawal J, Ratnayake G, Harridge S, Montgomery H, Hart N. Neuromuscular blockade and skeletal muscle weakness in critically III patients. Am J Resp Crit Care Med. 2012;185 (9);911-7.

10. Grimm A, Teschner U, Porzelius C, Ludewing K, Zielske J, Witte OW, et al. Muscle ultrasound for early assessment of critical illness neuromyopathy in severe sepsis. Critical Care. 2013;17:R227.

11. Mesejo A, Pérez-Sancho E, Moreno E. Clinical consequences of neuromuscular impairments in critically ill patients. Nutr Hosp. 2006;21(Suppl. 3):104-13.

12. Pati S, Bird SJ. Diagnosis and management of critical illness polyneuropathy and critical illness myopathy. Curr Treat Options Neurol. 2007:9(2):85-92.

13. Howard RS, Tan SV, Z'Graggen WJ. Weakness on the intensive care unit. Pract Neurol. 2008;8(5):280-95.

14. Weber-Carstens S, Deja M, Koch S, Spranger J, Bubser F, Wernecke KD, et al. Risk factors in critical illness myopathy during the early course of critical illness: a prospective observational study. Crit Care. 2010;14(3):R119.

15. Gorson KC. Approach to neuromuscular disorders in the intensive care unit. Neurocrit Care. 2005;3(3):195-212.

16. Tobar E, Abedrapo M, Godoy J, Romero C. Delirium postoperatorio. Una ventana hacia una mejoría de la calidad y seguridad en la atención de pacientes quirúrgicos. Rev Chil Cir. 2012;64(3):297-305.

17. Bennett S, Hurford WE. When Should Sedation or Neuromuscular Blockade Be Used During Mechanical Ventilation? Respir Care. 2011; 56(2):168-76.

18. Fan E, Shahid S, Kondreddi VP, Bienvenu OJ, Mendez-Tellez PA, Pronovost PJ, et al. Attributable mortality and length of stay of trauma-related complications: a matched. Monitoring Quality Indicators in Critical Patients. [Intenet]. Consultado el 26 de febrero de 2018. Disponible en: https://semicyuc.org/wp-content/uploads/2018/10/quality_indicators_update_2011.pdf

19. Schneider G. Muscle relaxants in the ICU. Anasthesiol Intensivmed Notfallmed Schmerzther. 2009;44(5):358-64.

20. Sandiumenge A, Anglés R, Martínez-Melgar JL, Torrado H. Use of neuromuscular blockers in the critical patient. Med Intensiva. 2008;32 (1):69-76

21. Greenberg SB, Vender J. The Use of Neuromuscular Blocking Agents in the ICU. Where Are We Now? Crit Care Med. 2013;41(5):1332-44.

22. Piraino T, Fan E. Acute life-threatening hypoxemia during mechanical ventilation. Curr Opin Crit Care. 2017;23(6):541-8.

23. Rios $F$, Iscar $T$, Cardinal-Fernández $P$. What every intensivist should know about acute respiratory distress syndrome and diffuse alveolar damage. Rev Bras Ter Intensiva. 2017;29(3):354-63.

24. Ruscic KJ, Grabitz SD, Rudolph MI, Eikermann M. Prevention of respiratory complications of the surgical patient: actionable plan for continued process improvement. Curr Opin Anaesthesiol. 2017;30(3):399-408.

25. De Backer J, Hart N, Fan E. Neuromuscular Blockade in the $21^{\text {st }}$ Century Management of the Critically III Patient. Chest. 2017;151(3):697-706.

26. Murray MJ, DeBlock H, Erstad B, Gray A, Jacobi J, Jordan C, et al. Clinical Practice Guidelines for Sustained Neuromuscular Blockade in the Adult Critically III Patient. Crit Care Med. 2016;44(11):2079-103.

27. Grawe ES, Bennett S, Hurford WE. Early Paralysis for the Management of ARDS. Respir Care. 2016;61(6):830-8.

28. Munshi L, Rubenfeld G, Wunsch H. Adjuvants to mechanical ventilation for acute respiratory distress syndrome. Intensive Care Med. 2016;42(5):775-8.

29. Claesson J, Freundlich M, Gunnarsson I, Laake JH, Møller $\mathrm{MH}$ Vandvik PO, et al. Scandinavian clinical practice guideline on fluid and drug therapy in adults with acute respiratory distress syndrome. Acta Anaesthesiol Scand. 2016;60(6):697-709. 
30. Hraiech S, Dizier S, Papazian L. The use of paralytics in patients with acute respiratory distress syndrome. Clin Chest Med. 2014;35(4):753-63.

31. Sottile PD, Albers D Moss MM. Neuromuscular blockade is associated with the attenuation of biomarkers of epithelial and endothelial injury in patients with moderate-to-severe acute respiratory distress syndrome. Crit Care. 2018; 22:63.

32. Przybysz TM, Heffner AC. Early Treatment of Severe Acute Respiratory Distress Syndrome. Emerg Med Clin North Am. 2016;34(1):1-14.

33. Simonis FD, Binnekade JM, Braber A, Gelissen HP, Heidt J, Horn J, PReVENT--protective ventilation in patients without ARDS at start of ventilation: study protocol for a randomized controlled trial. Trials. 2015;24:16:226.
34. Needham DM, Wozniak AW, Hough CL, Morris PE, Dinglas VD, Jackson JC, et al. Risk factors for physical impairment after acute lung injury in a national, multicenter study. Am J Respir Crit Care Med. 2014; 189(10):1214-24.

35. Spieth PM, Zhang $\mathrm{H}$. Pharmacological therapies for acute respiratory distress syndrome. Curr Opin Crit Care. 2014;20(1):113-21

36. Brogden TG, Bunin J, Kwon H, Lundy J, McD Johnston A, Bowley DM. Strategies for ventilation in acute, severe lung injury after combat trauma. J R Army Med Corps. 2015;161(1):14-21.

37. Díaz MC, Ospina-Tascón GA, Salazar CBC. Respiratory muscle dysfunction: a multicausal entity in the critically ill patient undergoing mechanical ventilation. Arch Bronconeumol. 2014;50(2):73-7. 